\title{
POLICONSUMO SIMULTÂNEO DE DROGAS ENTRE ESTUDANTES DE GRADUAÇÃO DA ÁREA DE CIÊNCIAS DA SAÚDE DE UMA UNIVERSIDADE: IMPLICAÇÕES DE GÊNERO, SOCIAIS E LEGAIS, SANTO ANDRÉ - BRASIL
}

\author{
Maria do Perpétuo Socorro de Sousa Nóbrega1, Laura Simich², Carol Strike ${ }^{3}$, Bruna Brands ${ }^{4}$, Norman \\ Giesbrecht ${ }^{5}$, Akwatu Khenti ${ }^{6}$
}

\begin{abstract}
${ }^{1}$ Ph.D. Doutora em Ciências da Saúde. Professora do Curso de Graduação e Pós-graduação em Enfermagem da Faculdade de Medicina do ABC (FMABC). Coordenadora da Educação Permanente em Enfermagem Psiquiátrica da Sociedade Paulista para Desenvolvimento da Medicina (SPDM). São Paulo, Brasil. E-mail: perpetuasn1@gmail.com

2 Ph.D. Researcher. Health Systems Research and Consulting Unit. Centre for Addiction and Mental Health. University of Toronto. Canada. E-mail: laura_simch@camh.net

${ }^{3}$ Ph.D. Researcher. Health Systems Research and Consulting Unit. Centre for Addiction and Mental Health. University of Toronto. Canada. E-mail: carol_strike@camh.net

${ }^{4}$ Ph.D. Researcher Senior. Office of Research and Surveillance, Drug Strategy and Controlled Substances Programme. Health Canada and Public Health and Regulatory Policies. Center for Addiction and Mental Health. University Toronto. Canada. E-mail: bruna_brands@camh.net

${ }^{5}$ Ph.D. Researcher. Health Systems Research and Consulting Unit. Centre for Addiction and Mental Health. University of Toronto. Canada. E-mail: norman_giebsbrecht@camh.net

${ }^{6}$ MSc. Director of Office of International Health. Center for Addiction and Mental Health (CAMH). Toronto, Canada. E-mail: akwatu_khenti@camh.net
\end{abstract}

RESUMO: Estudo transversal que teve por objetivo entender os padrões de policonsumo simultâneo de drogas e suas implicações de gênero, sociais e legais entre estudantes universitários. Participaram 275 estudantes de Ciências da Saúde de uma universidade em Santo André, Brasil. O policonsumo foi detectado em 27,9\% dos estudantes da amostra. Combinações: álcool + cannabis; álcool + medicamentos prescritos; álcool + tabaco + cannabis; tabaco + cannabis; e tabaco + medicamentos prescritos. As justificativas foram: ajudar a relaxar; perder inibições; permanecer acordado; ajudar a fazer algo que aborrece e melhorar o efeito de outras substâncias. Não se observou associação entre situações legais e sociais, os homens admitiram utilizar mais o policonsumo que as mulheres, a religião representou fator de proteção ao policonsumo, que foi inferior ao encontrado na literatura. Por se tratar de futuros profissionais de saúde, políticas institucionais devem ser discutidas no intuito de diminuir a exposição e vulnerabilidade destes às substâncias psicoativas.

DESCRITORES: Estudantes. Comportamento aditivo. Transtornos relacionados ao uso de substancias. Fatores de risco. Drogas ilícitas.

\section{SIMULTANEOUS POLYDRUGS USE AMONG UNDERGRADUATE STUDENTS OF HEALTH SCIENCES OF ONE UNIVERSITY: GENDER, SOCIAL AND LEGAL IMPLICATIONS, SANTO ANDRÉ - BRAZIL}

\begin{abstract}
Cross sectional study that aims to understand the patterns of polydrugs use and its gender, social and legal implications among undergraduate students. 275 undergraduate students from the Faculty of Health Sciences from one university in Santo André, Brazil, participated in the study. The simultaneous polydrug use was detected in $27.9 \%$ of the sample. Combinations: alcohol + cannabis; alcohol + prescription drugs; alcohol + tobacco + cannabis; tobacco + cannabis; and tobacco + prescription drugs. Reasons: To help relaxing, to lose inhibitions, to help staying awake, to help on doing something upsetting, and to improve the effect of other substances. There was no observed association between legal and social circumstances, men used more poly consumption than women, and religion represented a protective factor to simultaneous polydrug use, which was lower than described in the literature. Considering that subjects in the represent future health care professionals, institutional policies should be discussed in order to reduce exposure and vulnerability to these substances.
\end{abstract}

DESCRIPTORS: Students. Addictive behavior. Substance use-related disorders. Risk factors. Street Drugs. 


\title{
POLICONSUMO SIMULTÁNEO DE DROGAS EN ESTUDIANTES UNIVERSITARIOS DE PREGRADO DEL ÁREA DE CIENCIAS DE LA SALUD EN UNA UNIVERSIDAD: IMPLICACIONES DE GÉNERO, SOCIALES Y LEGALES, SANTO ANDRÉ - BRASIL
}

\begin{abstract}
RESUMEN: Estudio transversal que busca comprender los patrones de poli-consumo simultáneo de drogas y sus consecuencias de genero, sociales y legales entre estudiantes universitarios. 275 estudiantes de las ciencias de la salud de pregrado de una universidad en Santo André, Brasil participaran en el estudio. El policonsumo simultáneo estuvo presente en $27,9 \%$ de los estudiantes de la muestra. Combinaciones: alcohol + marihuana; alcohol + medicamentos de prescripción; alcohol + tabaco + cannabis; tabaco + cannabis; y tabaco + medicamentos de prescripción. Razones: Para relajarse, perder inhibiciones, mantenerse despierto, ayudar a hacer algo que genera molestia y mejorar el efecto de otras sustancias. No se observó asociación entre circunstancias jurídicas y sociales, los hombres presentaron mayor poli-consumo que las mujeres, y la religión se presentó como un factor de protección para el poli-consumo, que fue menor que la encontrada en la literatura. Dado que se trata de futuros profesionales de la salud, las políticas institucionales deben ser analizadas con el fin de reducir la exposición y la vulnerabilidad a estas sustancias psicoactivas.
\end{abstract}

DESCRIPTORES: Estudiantes. Conducta adictiva. Transtornos relacionados con el uso de substancias. Factores de riesgo. Drogas ilícitas.

\section{INTRODUÇÃO}

O uso de Substâncias Psicoativas (SPs) lícitas e/ou ilícitas por estudantes universitários tem sido tema amplamente discutido na literatura nacional e internacional, representando importante crise de saúde pública para algumas nações. ${ }^{1}$ O Policonsumo Simultâneo de Drogas (PCSD) é um subconjunto do policonsumo concorrente e refere-se à coingestão de diferentes SPs, ao mesmo tempo (ex: indivíduo que bebe álcool e fuma maconha em uma única sessão). Já o consumo concorrente diz respeito ao uso de diferentes SPs, em diferentes ocasiões (ex: indivíduo que bebe álcool em um dia e fuma maconha no dia seguinte).

São vários os fatores que aumentam a vulnerabilidade dos universitários ao uso SP, seja de forma combinada ou não. Entre estes estão a superação da fase de transição da escola para a universidade, o aumento da disponibilidade destas substâncias, o nível de aceitação pelos colegas e a participação em atividades sociais. ${ }^{2}$

O PCSD pode produzir maior intoxicação e aumentar o risco de acidentes, agregando mais prejuízo do que o uso de uma substância isolada. Estudo realizado com estudantes universitários de $1^{\circ}$ ano encontrou que $93 \%$ deles faziam uso de PCSD. A prevalência da combinação de álcool e medicamentos psicoativos prescritos foi de $6,9 \%$, sendo prevalente entre os rapazes. ${ }^{3}$ Outro estudo mostrou que a maioria dos adultos jovens estão sujeitos ao abuso de álcool, medicamentos prescritos, ou ambos, tanto de modo concomitante como simultâneo, e revelaram que $7 \%$ haviam combinado essas substâncias. ${ }^{4}$

Pesquisa desenvolvida na Austrália encontrou no álcool e tabaco a combinação mais realizada por um quarto dos estudantes, e um em cada vinte estudantes havia associado cannabis e anfetaminas ou cannabis e ecstasy, além de $1 \%$ terem utilizado opiáceos e cannabis na mesma ocasião e as razões apresentadas foram para melhor a performance/ desempenho acadêmico. Concluiu ainda que, os residentes no campus da faculdade são duas vezes mais susceptíveis ao PCSD que os residentes com seus familiares. Apontaram forte correlação entre queda da média escolar e potencial para aumento de PCSD. Em contra partida, estudantes com notas/ médias ' $\mathrm{A}$ ' apresentaram menor probabilidade. ${ }^{5}$

Estudo conduzido com 1.610 homens e 1.447 mulheres, do segundo ano, em dez universidades do Reino Unido, revelou que $34 \%$ dos participantes foram considerados PCSD. As razões significativas foram: prazer, $89 \%$ (masc.) e $92 \%$ (fem.); ansiedade, $17 \%$ (masc.) e $22 \%$ (fem.); aumento dos níveis confiança, $22 \%$ (masc.) e 33\% (fem.) e, por fim, hábito, $31 \%$ (masc.) e $22 \%$ (fem.). ${ }^{6}$ Pouco se sabe como as diversas substâncias são utilizadas e as razões envolvidas para esse padrão de consumo. Em princípio são para reforçar efeitos desejados, diminuir efeitos indesejados ou mesmo regular os efeitos de uma substância sobre a outra. ${ }^{7}$

Em relação às questões legais e sociais decorrentes do PCSD pouco tem sido discutido na literatura. Por extensão, é difícil identificar se tem havido aumento ou diminuição nas questões legais relacionadas com a utilização de SP nas universidades. Dados oficiais americanos aponta entre as conseqüências legais crimes e atos violentos cometidos no campus que não são comunicados às autoridades locais, em torno de $11,6 \%$ a $17,2 \%$; vandalismo $9,3 \%-10,5 \%$ e problemas com a administração escolar $1,9 \%-2,5 \% .8$

Dentre as consequências sociais, observam-se a desintegração familiar e/ ou da comunidade; carga social relacionadas com quadros de intoxicação, diminuição da capacidade cognitiva, comprometi- 
mento na participação e envolvimento em atividades escolares, contribuindo para mau desempenho acadêmico, prejuízo na capacidade em se preparar para exames e/ ou participação em aulas, absenteísmo, não conclusão escolar, suspensão e retenção, atitude descomprometida frente à escola e professores. Como também baixo desempenho acadêmico (22\%) e ausência às aulas (28\%); perda de memória (26\%), 13\% sofrimento por lesões e complicações na saúde, como náuseas e vômitos, $47 \%{ }^{9}$

Usou-se como modelo conceitual a Teoria Bio-Psicossocial, ${ }^{10}$ que compreende o fenômeno do PCSD como uma complexa interação de aspectos biológicos, psicológicos, sociais, culturais e espirituais.

Assim, esse estudo teve como objetivo identificar os padrões de policonsumo simultâneo de drogas e suas implicações legais, sociais e de gênero entre estudantes universitários de ciências da saúde.

\section{METODOLOGIA}

Estudo do tipo transversal que faz parte de um estudo multicêntrico. A população foi constituída por estudantes do $1^{\circ} \mathrm{e} 2^{\circ}$ anos dos cursos de graduação da instituição (estimado em 513 estudantes). Foram selecionados, aleatoriamente, quatro disciplinas obrigatórias de cada curso. A amostra foi constituída por estudantes de graduação dos cursos de Enfermagem (32), Medicina (116), Farmácia (39), Nutrição (45), Fisioterapia (30) e Terapia Ocupacional (13), matriculados em período integral ou parcial, com idade igual ou superior a 18 anos, cuja soma total representou 275 estudantes. A participação foi voluntária e realizada somente após assinatura do Consentimento Livre e Esclarecido (TCLE).

Para coleta dos dados utilizou-se um questionário anônimo auto aplicável construído pelos investigadores, no qual foram incorporadas algumas questões de instrumentos publicados na literatura, com a respectiva autorização dos autores, como: Wasting the best and the brightest: substance abuse at America's colleges and universities"; "Razões para o consumo de droga" ${ }^{\prime 11}$, Canadian Survey ${ }^{1}$ e CAGE. ${ }^{12}$ Ao final, o questionário constituiu-se por quatro seções, com 58 questões, abordando dados sócio-demográficos, utilização de substâncias psicoativas e conseqüências associadas. O questionário foi elaborado em inglês e passou pelo processo de "back translation", foi realizada análise fatorial, com adaptações prévias à coleta.

O processo de recrutamento foi conduzido pela investigadora principal e duas assistentes trei- nadas. A coleta transcorreu de forma tranquila e teve apoio dos professores e foi realizada nas salas de aulas, na ausência destes. Inicialmente foi explicado os objetivos, as características gerais do estudo e realizado leitura do TCLE, com espaço para dúvidas. A coleta durou em média 40 minutos. Foram destinadas duas caixas coletoras (questionários e outra para os TCLE assinados), garantindo maior sigilo.

As variáveis avaliadas foram: Implicações de gênero - papéis e expectativas sociais associadas a homens ou mulheres e as razões para o consumo de droga; Implicações sociais - experiências acadêmicas, familiares, trabalho, financeira ou sexual; Implicações legais - suspensões/problemas com a administração universitária/ polícia e o PCSD. Os dados foram analisados descritivamente, determinando a distribuição de frequência e as relações lógicas que aconteceram entre as variáveis do estudo. As variáveis categóricas foram analisadas aplicando-se a prova Qui-quadrado. As variáveis quantitativas foram analisadas aplicando-se a prova de correlação de Pearson. Considerou-se um nivel de confiança de $95 \%$. Os dados foram analisados pelo programa estatístico (SPSS) Versão 15.0. O estudo foi aprovado pelo Research Ethics Board (REB) of Centre for Addiction and Mental Health (CAMH-Prot. 225/2008) e pelo Comitê de Ética da Faculdade de Medicina do ABC da cidade de Santo André, São Paulo, Brasil (Prot. 312/2008).

\section{RESULTADOS}

\section{Características sóciodemográficas}

A tabela 1 mostra que 80,7\% dos estudantes são do sexo feminino e $90,1 \%$ solteiros, $85,4 \%$ residem com família de origem e $11,3 \%$ com outros parentes fora do campus, $28 \%$ exercem atividade remunerada 9,8\% integral e 18,9\% parcial, e 71,3\%, apenas estudam. O nível educacional dos pais varia de secundário 35,2\% (mãe) e 32,1\% (pai) e superior $51,5 \%$ (mãe) e $51,1 \%$ (pai). Para $39,6 \%$ a religião é importante, $37,5 \%$ muito importante e $23 \%$ pouco/não importante. Dos 275 estudantes, $42,2 \%$ são do curso de Medicina, $16,4 \%$ da Nutrição, $14,2 \%$ de Ciências Farmacêuticas, 11,6\% de Enfermagem, 10,9\% do curso de Fisioterapia e 4,75\% de Terapia Ocupacional. Frequentam o $1^{\circ}$ ano $49,5 \%$ e o $2^{\circ}$ ano $50,5 \%$. Média de idade de $22,15 \%$, oscilando de 18 e 51 .

Quanto à importância dada às atividades universitárias, $41,8 \%$ consideram as festas importantes, $42,9 \%$ as atividades esportivas, $38,9 \%$, as atividades artísticas, $47,6 \%$ a participação em organizações 
políticas, 50,9\% a participação em organizações estudantis, $43,2 \%$ a participação em organizações culturais/étnicas e $46,9 \%$ em atividades comunitárias. Atividades acadêmicas são importantes para $66,1 \%$ dos estudantes, e os clubes recreativos para $40,4 \%$ destes. Após terem iniciado a faculdade os estudantes apontam o relacionamento com os pais como muito bom $54,6 \%$ e bom $39,6 \%$.

As médias escolares foram consideradas ótimas por $62,7 \%$ e $56,2 \%$ estão satisfeitos com seu rendimento. As situações cotidianas nos últimos 12 meses foram: dificuldades financeiras $44,4 \%$; conflitos familiares $42,5 \%$; conflitos com parceiros $30,5 \%$; atividade sexual sem proteção $21,1 \%$; relações sexuais inesperadas/não planejadas 13,1\% e ausência frequente as aulas $8,7 \%$, comuns aos estudantes $1^{\circ} \mathrm{e} 2^{\circ}$ ano. As fontes de estresse cotidianas foram as tarefas escolares para $73,5 \%$ da amostra.

Tabela 1 - Características sóciodemográficas dos estudantes da amostra, Santo André - SP, 2009 $(\mathrm{n}=275)$

\begin{tabular}{|c|c|c|}
\hline Variáveis & $\mathrm{n}$ & $\%$ \\
\hline \multicolumn{3}{|l|}{ Sexo } \\
\hline Masculino & 53 & 19,3 \\
\hline Feminino & 222 & 80,7 \\
\hline \multicolumn{3}{|l|}{ Curso universitário } \\
\hline Medicina & 116 & 42,2 \\
\hline Enfermagem & 32 & 11,6 \\
\hline Farmácia & 39 & 14,2 \\
\hline Nutrição & 45 & 16,4 \\
\hline Fisioterapia & 30 & 10,9 \\
\hline Terapia Ocupacional & 13 & 4,7 \\
\hline \multicolumn{3}{|l|}{ Curso } \\
\hline Integral & 116 & 42,2 \\
\hline Parcial & 159 & 57,8 \\
\hline \multicolumn{3}{|l|}{ Trabalho } \\
\hline Integral & 27 & 9,8 \\
\hline Parcial & 52 & 18,9 \\
\hline Não trabalha & 196 & 71,3 \\
\hline \multicolumn{3}{|l|}{ Ano do curso } \\
\hline $1^{\text {a }}$ série & 136 & 49,5 \\
\hline $2^{\mathrm{a}}$ série & 139 & 50,5 \\
\hline \multicolumn{3}{|l|}{ Estado civil } \\
\hline Casado & 18 & 6,6 \\
\hline União livre & 4 & 1,5 \\
\hline Separado/divorciado & 5 & 1,8 \\
\hline Viúvo & - & - \\
\hline Solteiro & 246 & 90,1 \\
\hline \multicolumn{3}{|l|}{ Religião } \\
\hline Muito importante & 103 & 37,5 \\
\hline Importante & 109 & 39,6 \\
\hline Pouco importante & 48 & 17,5 \\
\hline Não é importante & 15 & 5,5 \\
\hline \multicolumn{3}{|l|}{ Moradia } \\
\hline Em casa com minha família & 234 & 85,4 \\
\hline Com outros parentes & 19 & 6,9 \\
\hline Fora da faculdade, com outros estudantes & 12 & 4,4 \\
\hline Fora da faculdade, sozinho & 8 & 2,9 \\
\hline Outros & 1 & 0,4 \\
\hline
\end{tabular}

Policonsumo simultâneo de substâncias por universitários

Observa-se na tabela 2, que o PCSD foi realizado por $27,9 \%$ dos estudantes. A combinação álcool + cannabis feita por 30\% últimos 12 meses, seguido da combinação de álcool + medicamentos prescritos de $22 \%$, a combinação de álcool + tabaco + cannabis feita por $16 \%$, a combinação de tabaco + cannabis feita por $8 \%$, e de tabaco + drogas prescritos por $8 \%$. Outras combinações feitas nos últimos 12 meses $42 \%$, álcool + tabaco $5,5 \%$; álcool + tabaco + inalantes $4 \%$, e PCSD de álcool + tabaco + drogas prescritas feito por $7 \%$.

Tabela 2- Distribuição dos estudantes com relação ao policonsumo simultâneo de substâncias psicoativas nos últimos 12 meses, Santo AndréSP, $2009(n=50)$

\begin{tabular}{lrrrrr}
\hline \multirow{2}{*}{ Drogas } & \multicolumn{2}{c}{ Masculino } & \multicolumn{2}{c}{ Feminino } \\
& \multicolumn{2}{c}{$\mathbf{n = 1 2}$} & \multicolumn{2}{c}{$\mathbf{n = 3 8}$} \\
\cline { 2 - 6 } & $\mathbf{n}$ & \multicolumn{1}{c}{$\mathbf{n}$} & \multicolumn{1}{c}{$\%$} \\
\hline Álcool + cannabis & 4 & 33,3 & 11 & $\mathbf{2 8 , 9}$ \\
Álcool + cocaína & 1 & 8,3 & - & - \\
Álcool + drogas prescritas & 4 & 33,3 & 7 & 18,4 \\
Álcool + tabaco + cannabis & 3 & 25,0 & 5 & 13,1 \\
Álcool + cocaína + tabaco & - & - & - & - \\
Álcool + cocaína + cannabis + & - & - & - & - \\
tabaco & & & & \\
Cannabis + cocaína & 1 & 8,3 & - & - \\
Cannabis + drogas prescritas & - & - & - & - \\
Tabaco + cannabis & 1 & 8,3 & 3 & 7,8 \\
Tabaco + drogas prescritas & 1 & 8,3 & 3 & 7,8 \\
Outras combinações & 5 & 41,6 & 16 & 42,1 \\
\hline
\end{tabular}

\section{Razões para policonsumo de substâncias}

De acordo com a tabela 3, observa-se as razões pelas quais os estudantes fazem PCSD, e estão subdivididas em cinco aspectos centrais: mudança de humor, efeitos físicos, propósitos sociais, facilitar atividade e manejar efeitos de outras substâncias.

No aspecto mudança de humor, o critério "ajudar a relaxar" foi a razão mais apontada pelos estudantes para utilizar mais de uma substância ao mesmo tempo $74 \%$. Com relação aos efeitos físicos, o critério "Ajudar a permanecer despertado/acordado" representou $32 \%$ das razões citadas. Nos propósitos sociais, "Ajudar a perder inibições" foi a razão mais citada $58 \%$. No aspecto facilitar atividades, "Ajudar a fazer algo que aborrece" foi apontado como o principal motivo por $12 \%$ dos estudantes. 
No aspecto manejar efeitos de outras substâncias, "Melhorar o efeito de outras substâncias" foi a razão apontada por $10 \%$ de dos estudantes.

A frequência de PCSD em grupo é alta 79,6\%, tanto que $64,1 \%$ apontaram que todos do grupo de amigos decidem o que vão consumir, 10,3\% recebem influência de amigos e 33,3\% acredita que resolvem por si o que vão utilizar. Os estudantes acreditam que os homens influenciam mais as mulheres ao PCSD $47 \%$ do que o inverso $38,7 \%$.Os lugares para PCSD se dividem em casas noturnas $70 \%$ e festas no campus $38 \%$. Os dias escolhidos são sábados $97,4 \%$ e sextas $66,7 \%$. Adquirem dro- gas no campus $28 \%$, e medicamentos controlados adquirem com amigos $33,5 \%$, revendedores $11,6 \%$, família $6,5 \%$, farmácias caseiras $7,3 \%, 66 \%$ adquirem com o próprio dinheiro e $30 \%$ dinheiro da família. Os estudantes opinam que o uso de drogas no campus é problema muito importante $46,5 \%$. Para $76,2 \%$ o uso por profissionais da saúde é um grande problema, $56,3 \%$ sentem-se culpados pelo PCSD e $43,7 \%$ não percebem problema. Nenhum estudante manifestou que sente necessidade de fazer PCSD pela manhã, para despertar ou controlar os nervos, ou para aliviar o mal-estar das substâncias psicoativas.

Tabela 3 - Distribuição dos estudantes e razões para policonsumo de substâncias, Santo André-SP, $2009(n=50)$

\begin{tabular}{llrr}
\hline Aspecto central & Razões & $\mathbf{n}$ & \% \\
\hline \multirow{4}{*}{ Mudança de humor } & Sentir melhor quando deprimido & 22 & 44,0 \\
& Parar de se preocupar com problemas & 19 & 38,0 \\
& Ajudar a relaxar & 37 & 74,0 \\
& Sentir emoção ou euforia & 18 & 36,0 \\
& Sentir drogado ou intoxicado & 1 & 2,0 \\
& Melhor as sensações no sexo & 7 & 14,0 \\
& Permanecer desperto/acordado & 16 & 32,0 \\
Efeitos físicos & Ajudar a perder peso & 4 & 8,0 \\
& Ajudar a dormir & 12 & 24,0 \\
Propósitos sociais & Ajudar a curtir a companhia dos amigos & 26 & 52,0 \\
& Sentir confiança em situação social & 21 & 42,0 \\
& Ajudar a perder inibições & 29 & 58,0 \\
& Manter-se bem numa saída noturna & 21 & 42,0 \\
Facilitar atividade & Relacionar melhor devido pressão social & 8 & 16,0 \\
& Concentrar no trabalho ou estudo & 5 & 10,0 \\
Manejar efeitos de outras substâncias & Incrementar atividades (esporte/jogos) & 4 & 8,0 \\
& Ajudar a fazer algo que aborrece & 6 & 12,0 \\
& Aelhorar efeitos de outras substâncias & 5 & 10,0 \\
& Atenuar efeitos de outras substâncias & 4 & 8,0 \\
\hline
\end{tabular}

\section{Policonsumo de substâncias e situações cotidianas}

Entre as situações cotidianas consultadas e que podem ter influenciado o PCSD (Tabela 4) ter sido ferido/lesionado por outras pessoas foi a mais apontada $5,5 \%$, seguido por ter sofrido acidente automobilístico, ter tido problemas com a polícia e problemas com a administração da faculdade foram relevantes nos últimos 12 meses. Não foi encontrada associação significativa entre situações cotidianas e PCSD.
Poucos estudantes sofreram ato violento quando sob a influência de alguma substância $5,6 \%$, mas todos que sofreram relataram que o agressor estava sob efeito delas. Recomendam que a Faculdade deve providenciar serviços de aconselhamento $72,4 \%$, desenvolver clubes universitários/grupos de apoio 70,2\%, estimular políticas universitárias relacionadas ao tema $67,3 \%$, atividades de educação específica/ seminários ou conferências acerca das substâncias psicoativas, $60,4 \%$. 
Tabela 4 - Distribuição de estudantes, segundo o policonsumo, situações cotidianas e gênero, Santo André-SP, 2009

\begin{tabular}{|c|c|c|c|c|c|}
\hline \multirow{3}{*}{ Situações experimentadas nos últimos 12 meses } & \multicolumn{4}{|c|}{$\begin{array}{l}\text { Uso de mais de uma substância psicoativa ao } \\
\text { mesmo tempo, nos últimos } 12 \text { meses }\end{array}$} & \multirow{3}{*}{ p-valor } \\
\hline & \multicolumn{2}{|c|}{ Não } & \multicolumn{2}{|c|}{ Sim } & \\
\hline & $n=129$ & $(\%)$ & $\mathrm{n}=50$ & $(\%)$ & \\
\hline Ausentar-se freqüentemente das aulas & 13 & $(10,1)$ & 7 & $(14,0)$ & 0,629 \\
\hline Atividade sexual sem proteção & 30 & $(23,3)$ & 13 & $(26,0)$ & 0,848 \\
\hline Relações sexuais inesperadas/não planejadas & 16 & $(12,4)$ & 12 & $(24,0)$ & 0,091 \\
\hline Conflitos familiares & 61 & $(47,3)$ & 25 & $(50,0)$ & 0,873 \\
\hline Conflitos com o parceiro (a) & 41 & $(31,8)$ & 17 & $(34,0)$ & 0,915 \\
\hline Dificuldades financeiras & 63 & $(48,8)$ & 19 & $(38,0)$ & 0,254 \\
\hline Brigas que resultaram em prisão & - & - & 1 & $(2,0)$ & 0,279 \\
\hline Multas por dirigir sob efeito de álcool & 3 & $(2,3)$ & - & - & 0,561 \\
\hline Acidentes automobilísticos & 5 & $(3,9)$ & 2 & $(4,0)$ & 1,000 \\
\hline Encarceramento/prisão & 1 & $(0,7)$ & - & $(0,0)$ & 1,000 \\
\hline Ferido/lesionado por outras pessoas & 8 & $(6,2)$ & 4 & $(8,0)$ & 0,741 \\
\hline Outros problemas com a polícia & 1 & $(0,7)$ & 1 & $(2,0)$ & 0,482 \\
\hline Repreensão acadêmica & - & - & - & - & 1,000 \\
\hline Suspensão da faculdade & - & - & 1 & $(2,0)$ & 0,279 \\
\hline Outros problemas com administração da faculdade & 2 & $(1,5)$ & 3 & $(6,0)$ & 0,134 \\
\hline \multicolumn{6}{|l|}{ Sexo } \\
\hline Masculino & 22 & $(17,1)$ & 12 & $(24,0)$ & \multirow{2}{*}{0,395} \\
\hline Feminino & 107 & $(82,9)$ & 38 & $(76,0)$ & \\
\hline
\end{tabular}

*Teste qui-quadrado de Pearson; +Teste Exato de Fisher.

instituição privada, localizada no sudeste do Brasil

\section{Policonsumo de substâncias e religião}

A relação entre a variável religião e o PCSD nos últimos 12 meses (Tabela 5) mostrou uma associação significativa $(p=0,011)$. Estudantes que não consumiram substância psicoativa consideram a religião muito importante $30,2 \%$ e importante $48,1 \%$.

Tabela 5 - Distribuição de estudantes segundo policonsumo nos últimos 12 meses e importância da religião. Santo André-SP, 2009

\begin{tabular}{|c|c|c|c|}
\hline \multirow{3}{*}{$\begin{array}{l}\text { Importância da } \\
\text { religião/crenças } \\
\text { religiosas }\end{array}$} & \multicolumn{2}{|c|}{$\begin{array}{c}\text { Policonsumo - últimos } \\
12 \text { meses }\end{array}$} & \multirow{3}{*}{ p-valor* } \\
\hline & Não & Sim & \\
\hline & $n=129(\%)$ & $n=50(\%)$ & \\
\hline Muito importante & $39(30,2)$ & $12(24,0)$ & \\
\hline Pouco importante & $62(48,1)$ & $16(32,0)$ & 0,011 \\
\hline Muito importante & $28(21,7)$ & $22(44,0)$ & \\
\hline
\end{tabular}

*Teste qui-quadrado de Pearson.

\section{DISCUSSÃO}

Nessa pesquisa exploratória foi possível identificar o perfil sóciodemográfico de uma amostra intencional de 275 universitários de uma e aplicar os conceitos de implicações de gênero, sociais e legais, relacionados ao PCSD. A média de idade é de $22,15 \%$, maioria feminina, solteiros, trabalhadores e residentes com família de origem. Encontrou-se que $72,7 \%$ dos estudantes que usaram drogas na vida o fizeram com 16,2 anos, destes 27,9\% fizeram PCSD com média de idade de 17,8 anos, mostrando um intervalo curto de 1,5 anos. O estudo identificou que o PCSD foi inferior aos $93 \%$ encontrado na literatura internacional ${ }^{3} \mathrm{e}$ nacional de $43 \% .{ }^{13}$

Ainda que de forma concomitante, o álcool manteve a posição de substância mais utilizada, seguido de tabaco, das drogas psicoativas prescritas, cannabis e inalantes. Quanto ao uso simultâneo, predominaram as associações tradicionais de álcool + cannabis, álcool + medicamentos prescritos, ál$\mathrm{cool}+$ tabaco + cannabis, tabaco + cannabis e tabaco + medicamentos prescritos, e em alguns casos de álcool + tabaco; álcool + inalantes; álcool + tabaco + inalantes; álcool + tabaco + drogas prescritas.

A combinação álcool + cannabis foi superior aos $7 \%$ encontrado em recente survey americano ${ }^{14}$ e inferior aos $36 \%$ na vida encontrado em estudo nacional, ${ }^{13}$ sendo considerado um padrão freqüentemente realizado pela população geral, quando feita aumenta a absorção de THC, intensificando seus efeitos subjetivos, prejudicando as 
funções motoras e intelectuais ${ }^{15}$ e a dependência da substância em si. ${ }^{16-17}$ Esse padrão geralmente está associado a expectativa do usuário, quanto a melhora de humor/sentimento negativo. ${ }^{11}$

A combinação de álcool + medicamentos prescritos de $22 \%$ nos últimos 12 meses foi considerada elevada frente aos $7 \%$ encontrados na literatura, ${ }^{2,4}$ sendo especialmente perigosa pois desencadeia vários efeitos cumulativos, além de casos letais decorrentes da co-ingestão. ${ }^{18-20} \mathrm{~A}$ combinação de álcool + tabaco + cannabis 16\%, está entre as substâncias comumente mais utilizadas por universitários. ${ }^{7} \mathrm{O}$ hábito de usar tabaco está associado ao aumento de 4,5 vezes para consumo, abuso e dependência de cannabis $^{21}$, a combinação feita foi realizada por $8 \%$ dos estudantes da amostra.

Associação de tabaco + drogas prescritas por $8 \%$ não surpreende, já que são substâncias consumidas diariamente, especialmente o tabaco, para àqueles que são dependentes e mesmo para os medicamentos prescritos, para aqueles que usam sob algum critério médico. Possivelmente em busca de equilíbrio pelos efeitos das substâncias, os estudantes associaram álcool + tabaco + drogas prescritas, ou seja, alternaram substância estimulante como o tabaco e depressoras. Mesmo com pouca expressão da associação de álcool + inalantes e álcool + tabaco + inalantes, sabe-se que o uso de inalantes é bastante difundido entre os estudantes universitários brasileiros.

As razões para PCSD em todos os aspectos explorados foram inferiores aos encontrados na literatura ${ }^{11,13}$ e estão compreendidas dentro dos aspectos biológicos, psicológicos, sociais, espirituais e culturais, de acordo com a Teoria Bio-Psicossocial. ${ }^{10}$ Houve predomínio da necessidade de combinação para lidar com situações sociais, facilitar a realização de atividades pouco prazerosas e perder as inibições naturais da idade. A combinação e o conjunto dessas razões apontam para a necessidade de medidas que sirvam de suporte para lidar com os estressores cotidianos, como também despertar sensações que tragam bem estar geral. A associação com o propósito de manejar efeitos de outras substâncias foi inexpressiva.

É possível que o fato de não encontrar associações significativas entre situações cotidianas e PCSD seja devido às limitações da amostra, mais do que a falta de relação entre as variáveis. Por se tratar de estudantes de $1^{\circ}$ e $2^{\circ}$ ano, fase de transição/adaptação a um novo cenário, que estão recebendo constantemente influências de novos modelos sociais, com repertório comportamental escasso para enfrentamento, esses jovens apresentam-se vulneráveis, ${ }^{22-23}$ às influências, tanto de forma direta, por meio da oferta, incentivo a compra, encorajamento ao uso, como indireta, no compartilhamento de normas que valorizam e reforçam o uso dessas substâncias.

Quanto à questão gênero os homens fizeram mais uso que as mulheres. Os estudantes acreditam que o uso não é bom para ambos e que os homens influenciam mais as mulheres do que o contrário, representação que reflete o modelo de várias culturas, nas quais as mulheres são mais vulneráveis às influências dos amigos/parceiros amorosos, e vem carregada do simbolismo das relações de poder entre os sexos. ${ }^{24-25}$ Julgar a religião pouco importante ou não importante representou um fator colaborativo ao consumo. A religião tem sido considerada como fator protetor ao uso de drogas, pois estabelece controle por meio de normas, padrões de socialização, ética e moral, veiculados pelas instituições religiosas. ${ }^{11}$

Os estudantes apontaram as tarefas escolares como a principal fonte de estresse, o PCSD foi utilizado em parte para obter relaxamento físico e mental, estando associado a excessiva agenda escolar, as cobranças do curso, na expectativa de permanecer despertado/acordado para lidar com essas exigências, sendo a substância adquirida e usada, em alguns casos, dentro do campus. Em contrapartida escolhem como dias preferidos as sextas-feiras e os sábados, demonstrando que o uso não é feito apenas com o propósito de lidar com problemas do cotidiano, mas em ocasiões sociais/ festivas, provavelmente em grupo.

Apesar das dificuldades financeiras uma parte da amostra é constituída por trabalhadores e adquirem drogas com o próprio dinheiro. Por se tratarem de estudantes da área da saúde, acredita-se ainda que o PCSD possa ser realizado com o intuito de lidar com sentimentos de angústia por saberem que vão lidar com a dor e sofrimento de seus pacientes, pela crise natural da idade e muitas vezes devido a precoce entrada na universidade, que pode vir acompanhada de incertezas quanto a escolha profissional.

Não foi verificada relação entre consequências legais/sociais e o PCSD e poucos estudantes se envolveram em situações de risco. No entanto, aqueles que passaram por essa situação, foram vitimados por pessoas sob efeito de SP. Na percepção dos estudantes o uso de drogas no campus da faculdade representa problema muito importante 
os estudantes consideram problema o uso por profissionais da saúde. Situam o compromisso institucional na prevenção ao uso de drogas por seus discentes, apontando que esta deve providenciar serviços de aconselhamento e estimular políticas universitárias relacionadas com atividades educativas específicas.

\section{CONCLUSÃO}

Esse estudo é o primeiro do gênero realizado na especifica universidade e explora uma nova linha de conhecimento sobre um dos problemas que mais afetam nossa sociedade. O fenômeno do PCSD pode diminuir a tolerância de profissionais com as drogas, banalizando sua atuação no aconselhamento sobre as conseqüências deletérias aos seus futuros clientes. Sugere-se espaço para discussão, desenvolvimento de políticas internas, que diminuíam a vulnerabilidade dos estudantes. Essas informações devem ser divulgadas e utilizadas em programas de prevenção em outros cenários educacionais.

\section{Limitações do estudo}

As limitações da amostra se somam as limitações para generalizar nossas conclusões, dada a especificidade da população estudada. Comparações com estudos internacionais são difíceis devido as diferenças de contexto.

\section{Recomendações}

Realização de estudos por outras instituições de ensino superior no Brasil para compreender melhor as expectativas dessa clientela.

\section{AGRADECIMENTOS}

Ao governo do Canadá/DFAIT, Organização dos Estados Americanos (OEA), Comissão Interamericana para o Controle e Abuso de Drogas (CICAD), Centre for Addiction and Mental Health (CAMH-Toronto/Canadá), estudantes e colaboradores. Um agradecimento especial ao Dr. Gustavo Mery, CAMH Reviewer, por sua colaboração na revisão e editoração final de todos os trabalhos e informes do grupo III.

\section{REFERÊNCIAS}

1. National Centre on Addiction and Substance Abuse. "Wasting the best and the brightest: Substance abuse at America's colleges and universities" [CASA].
New York, Columbia University; March; 2007. Disponível em: www.casacolumbia.org.

2. McCabe SE, Cranford JA, Morales M, Young A. Simultaneous and concurrent poly-drug use of alcohol and prescription drugs: prevalence, correlates, and consequences. J Stud Alcohol. 2006 Jul; 67(4):529-37.

3. Martin CS, Clifford PR, Clapper RL. Patterns and predictors of simultaneous and concurrent use of alcohol, tobacco, marijuana, and hallucinogens in first-year college students. J Subst Abuse. 1992; 4(3):319-26.

4. Earleywine M, Newcomb MD. Concurrent versus simultaneous polydrug use: Prevalence, correlates, discriminate validity, and prospective effects on health outcomes. Exp Clin Psychopharmacol. 1997 Nov; 5: 353-64.

5. Ashton E. Alcohol abuse makes prescription drug abuse more likely. National Institute of drugs abuse 2008 [acesso 2008 Jul 15]; 21(5). Disponível em: http://www.drugabuse.gov/NIDA_notes/ NNvol21N5/alcohol.html

6. O'Reily BO, Jessen J. Cannabis use among Australian tertiary students. South Pacific Journal of Psychology. 2005; 6(1):18-29.

7. Webb E, Ashton CH, Kelly P, Kamali F. Alcohol and drug use in UK university student. Lancet. 1996 Oct 5; 348(9032):922-5.

8. Barrett S, Darredeau C, Pihl R. Patterns of simultaneous poly-substance use in drug using university students. Hum Psychopharmacol. 2006 Jun; 21(4):255-63.

9. Engs R, Hansen DJ. Boozing and brawling on campus: a national study of violent problems associated with drinking over the past decade. J Crim Justice. 1994; 22(2):171-80.

10. Huyse F, Lyons J, Stiefel F, Slates J, Jonge P, Latour, C. Operationalizing the biopsychosocial model: the Intermed. Psychosomatics. 2001 Jan-Feb; 42(1):5-13.

11. Klingemann H, Gmel G. Introduction: social consequences of alcohol - the forgotten dimention? In: Klingemann H, Gmel G, editors. Mapping the social consequences of alcohol consumption. Dortrecht: Kluwer Academic Publishers; 2003. p.1-9.

12. Erwing J. Detecting alcoholism: the CAGE questionnaire. JAMA. 1984 Oct 12; 252(14):1905-7.

13. Brasil. Presidência da República. Secretaria Nacional de Políticas sobre Drogas. I Levantamento Nacional sobre o uso de álcool, tabaco e outras drogas entre universitários das 27 capitais brasileiras. Brasília: SENAD; 2010.Adlaf EM, Demers A, Gliksman L, editors. Canadian Campus Survey 2004. Toronto (CA): Centre for Addiction and Mental Health; 2005.

14. Midanik LT, Tam TW, Weisner C. Concurrent and simultaneous drug and alcohol use: results of the 2000 national Alcohol Survey. Drug Alcohol Depend. 2007 Sep 6; 90(1):72-8 
15. Staines GL, Magura S, Foote J, Deluca A, Kosanke N. Polysubstance use among alcoholics. J Addict Dis. 2001; 20(4):53-69.

16. Margolese HC, Malchy L, Negrete JC, Tempier R, Gill K. Drug and alcohol use among patients with schizophrenia and related psychoses: levels and consequences. Schizophr Res. 2004 Apr 1; 67(23):157-66.

17. Lukas S, Orozco S. Ethanol increases plasma Delta (9)-tetrahydrocannabinol (THC) levels and subjective effects after marihuana smoking in human volunteers. Drug Alcohol Depend. 2001 Oct 1; 64(2):143-9.

18. Ensslin JC. Combination of alcohol, drugs killed CSU Freshman. Rocky Mountain News. 2004 Dec 30 [acesso 2005 Apr 24]. Disponível: http:/ / www. rockymountainnews.com.

19. Leinwand D. Prescription abusers not just after a high. USA Today. 2005 May 25 [acesso 2005 Jun 10]. Disponível em: http:/ / www.usatoday.com/news/ health/2005-05-25-drugs_x.htm.
20. Petrillo L, Cantlupe J. Painkiller linked to 3 local deaths. San Diego Union Tribune. 2005 Feb 19 [acesso 2005 Mar 02]. Disponível: http://www. signonsandiego.com/uniontrib //news_7m19drug. html.

21. Gossop M, Manning V, Ridge G. Concurrent use of alcohol and cocaine: differences in patterns of use and problems among users of crack cocaine and cocaine powder. Alcohol Alcohol. 2006 Mar-Apr; 41(2):121-5.

22. Read J, Wood M, Davidoff O, Mclacken J, Campbell J. Making the transition from high school to college: the role of alcohol related a social influence factor in student's drinking. Subst Abus. 2002 Mar; 23(1):53-65.

23. Nóbrega MPSS, Oliveira EM. Alcoolismo feminino: dando voz às mulheres. Acta Paul Enferm. 2003 JulSet; 16(3):71-80.

24. Nóbrega MPSS, Oliveira EM. Mulheres usuárias de álcool: análise qualitativa. Rev Saúde Pública. 2005 Out; 39(5):816-23. 